\title{
Critical Current Degradation in HTS Wires Due to Cyclic Mechanical Strain
}

\author{
David T. Ryan, Liang Li, Xianrui Huang, J. W. Bray, Evangelos T. Laskaris, Kiruba Sivasubramaniam, \\ Aniruddha D. Gadre, James M. Fogarty, E. J. Harley, A. Otto, and A. Den Ouden
}

\begin{abstract}
HTS wires, which may be used in many devices such as magnets and rotating machines, may be subjected to mechanical strains from electromagnetic, thermal and centripetal forces. In some applications these strains will be repeated several thousand times during the lifetime of the device. We have measured critical current degradation due to repeated strain cycles for both compressive and tensile strains. Results for BSCCO-2223 HTS conductor samples are presented for strain values up to $0.5 \%$ and cycle numbers up to and beyond $10^{4}$.
\end{abstract}

Index Terms-Critical current degradation, cyclic strains, electric devices, HTS wires.

\section{INTRODUCTION}

$\mathbf{O}$ NE of the key factors in designing electrical machines using HTS wires is the $\mathrm{I}_{\mathrm{C}}$ (critical current) degradation due to mechanical strains. The $\mathrm{I}_{\mathrm{C}}$ of aHTS wire is degraded if it experiences sufficient mechanical strain. In the 100 MVA HTS generator that is currently under development in GE, the HTS wire in the rotor coil will experience both tensile and compressive strain due tocentrifugal loading and differential thermal contraction during cooldown/warm-up of the rotor. The HTS coil must survive hundreds to thousands thermal and start-up loading cycles.

There are many experiments and reports on $\mathrm{HTS} \mathrm{I}_{\mathrm{c}}$ degradation by mechanical strains [1]-[4]. Most of them investigate monotonic tensile strains. We conducted a series of $I_{c}$ degradation experiments to investigate the wire $\mathrm{I}_{\mathrm{c}}$ degradation under both tensile and compressive strains. Measurement of $\mathrm{I}_{\mathrm{c}}$ as a function of the number of strain cycles at different strain levels enables the fatigue behavior of the HTS wire to be determined. The degradation of $I_{c}$ due to cyclic strain has been measured by a number of groups. Data measured by GE, AMSC, and the University of Twente are presented here.

\section{Materials, ApPARATUS AND Definitions}

\section{A. Materials}

The measurement samples are silver/alloy BSCCO-2223 HTS tape with stainless steel reinforcement manufactured

Manuscript received October 9, 2004. This work was supported in part by the U.S. Department of Energy under Grant DE-FC36-02GO11100.

D. T. Ryan, L. Li, X. Huang, J. W. Bray, E. T. Laskaris, K. Sivasubramaniam, and A. D. Gadre are with General Electric Global Research Center, Niskayuna, NY 12309, USA (e-mail: li@crd.ge.com).

J. M Fogarty is with General Electric Energy, Schenectady, NY, USA.

E. J. Harley and A. Otto are with American Superconductor, Westborough, MA.

A. Den Ouden is with the Low Temperature Division, University of Twente, 7500 AE Enschede, The Netherlands.

Digital Object Identifier 10.1109/TASC.2005.849392
TABLE I

THE BSCCO-2223 HTS TAPE FROM AMSC

\begin{tabular}{l|l}
\hline Ic $(77 \mathrm{~K})$ & $124-138 \mathrm{~A}$ \\
\hline Thickness (average) & $0.292 \mathrm{~mm}$ \\
\hline Width (average) & $4.928 \mathrm{~mm}$ \\
\hline
\end{tabular}

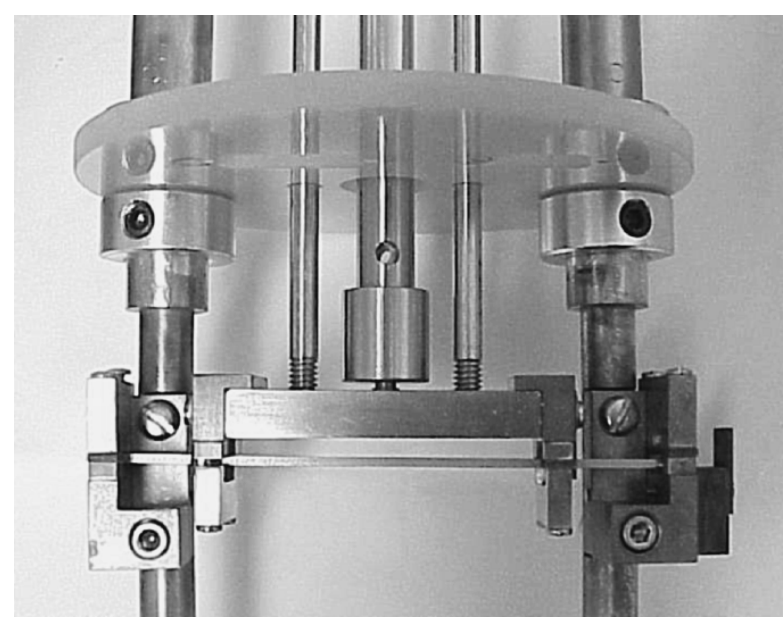

Fig. 1. The GE four-point bending apparatus. The copper rods on the sides are fixed in position, while the stainless steel rods at the center move up and down to bend the substrate by a programmable electric actuator located at the top of the apparatus but not shown in this figure. Voltage and current leads are flexible copper wires.

by AMSC using the oxide-powder-in-tube (OPIT) technique. Samples were selected from different batches of the HTS wire ordered from AMSC for the 100 MVA HTS generator development. Tape specifications are given in Table I.

\section{B. Measurement Apparatus and Procedure}

Measurements have been made using three different facilities located at GE, AMSC and the University of Twente. In all the facilities, the sample of HTS wire is bonded to one face of a stainless steel substrate, and the cyclic strain is applied by bending and straightening the substrate. The GE facility uses a four-point bending apparatus to do this as shown in Fig. 1. The bending device is controlled by an electric actuator, which pushes the center rods down to a predefined displacement. This four-point bending arrangement gives uniform bending and thus a uniform strain over the length of sample between the inner bending points. The set-up enables the determination of $I_{C}$ in high strain range $( \pm 1 \%)$, depending on the thickness of the substrate and the bending displacement at the center. Critical current is measured at $77 \mathrm{~K}$ using the $0.1 \mu \mathrm{V} / \mathrm{cm}$ criterion with voltage taps spaced $5 \mathrm{~cm}$ apart. The procedure is automated by 


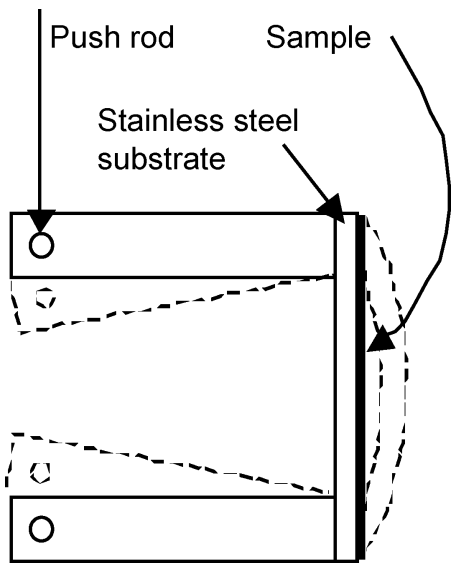

Fig. 2. Schematic of a bending spring.

a programmable power supply with a $2 \mathrm{~A}$ current step. The $\mathrm{I}_{\mathrm{C}}$ is determined by a simple interpolation. The sample strain is monitored by a strain gauge mounted on the surface of the HTS wire. To obtain the relation between $\mathrm{I}_{\mathrm{c}}$ and the number of strain cycles $\mathrm{N}$, the cyclic loading was stopped after a certain number of cycles and a V-I measurement was carried out at the predefined peak strain and at zero when the strain was relieved.

AMSC and Twente use a 'bending spring' apparatus similar to that shown in Fig. 2 and described in [1]. In this type of apparatus, the bending is not uniform over the sample, so only a short length $(1 \mathrm{~cm})$ is used for $I_{C}$ measurement.

\section{Definitions}

\section{Strain limits:}

We define failure of the wire as the point where $I_{c}$ retention falls below $95 \%$. This type of test may underestimate the magnitude of the strain to failure, since the $5 \%$ loss of $I_{c}$ may be due to a small fraction of the filaments near the outer surface of the wire that experience a higher strain than the rest of the wire. For data analysis the strain is taken at the mid-plane of the HTS wire.

Zero strain: the strain condition of a wire at $77 \mathrm{~K}$ after cooling without mechanical restraint. During measurements this condition is approximated by bonding the wire to a stainless steel substrate. (The CTE of stainless steel is well matched to that of the wire.)

Cyclic strain: mechanical strain applied to the HTS wire by repeated, monotonic transitions between two strain values. The wire is maintained at a constant temperature during the cycling. A single strain cycle consists of a transition from the start point to the end point and back to the start.

\section{RESULTS}

Results to date are plotted in Fig. 3, which shows $I_{c}$ retention under tensile and compressive cyclic strains. To determine the strain limits corresponding to $5 \% \mathrm{I}_{\mathrm{c}}$ degradation, the measurements were carried out focusing on the tensile and compressive strains at which the $5 \% \mathrm{I}_{\mathrm{c}}$ degradation was observed. These strains are around $-0.15 \%$ and $0.38 \%$ (negative values denoting compression). The open symbols in the figure represent retention after 100 cycles, and the shaded symbols represent satura-

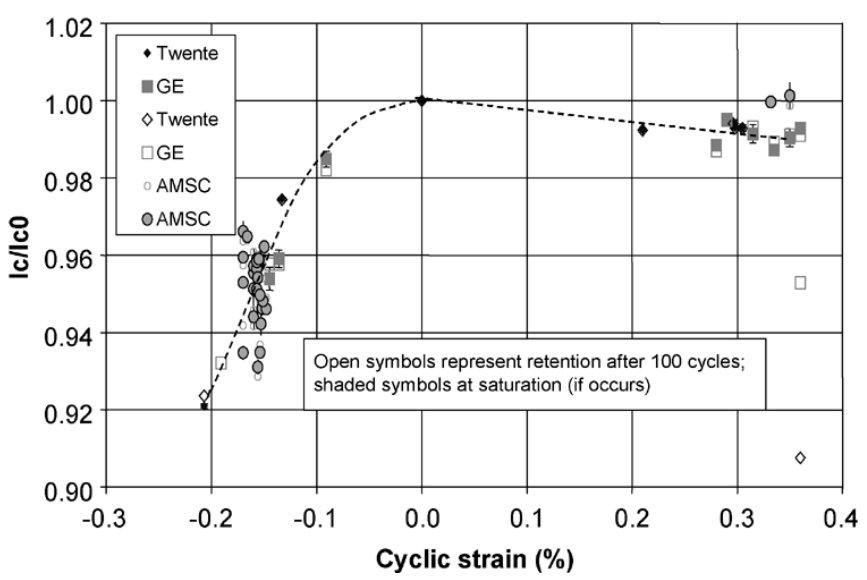

Fig. 3. Ic retention under tensile and compressive cyclic strain.

tion, which means no further degradation is observed when the number of cycles is increase more than 1000 . Typically, saturation was observed after 100-300 cycles, as we shall discuss in next sections. All of the runs at GE continued to $10^{4}$ cycles; most runs at Twente continued to $10^{3}$ cycles. The error bars indicate \pm one standard deviation of the $I_{c}$ values after saturation. As shown in the figure, the behavior of the tape under compressive and tensile cycles is different, and we shall therefore discuss them separately in more detail.

\section{A. Tensile Strain}

The behavior of the HTS tape under tensile strain cycles is as shown on the right hand side in Fig. 3. Up to a strain of $0.36 \%$, the $\mathrm{I}_{\mathrm{c}}$ remains almost constant or has very small reduction. Larger strain results in a dramatic reduction of $\mathrm{I}_{\mathrm{C}}$ to lower than 0.92 at $0.38 \%$ strain. This suggests that the peak tensile strain that the wire will experience in any application should remains lower than $0.35 \%$ with an adequate margin when such an HTS conductor is used.

The retention of the $I_{C}$ within tensile strain cycles of different magnitudes is shown in Fig. 4. The square dots are the data measured at the peak strain of the cycle, while the diamond dots were measured after the strain was relieved to zero. As expected, the reduction is larger at peak strain than at zero. These results are similar to those described of many BSCCO/Ag conductors without stainless steel reinforcement [1], [2]. In the strain range between 0.2 to $0.35 \%$, we found that the degradation saturated by 100 cycles. This indicates that, if we meet specifications for 100 cycles, we automatically meet the less stringent spec at $10^{4}$ cycles.

A total of 19 samples were measured under tensile strain cycles. The critical strain follows a normal distribution. The mean value and the standard deviation were calculated as shown in Fig. 5.

To analyze the variation of the HTS wire batch to batch, samples were randomly selected from 4 different spools of the wire from AMSC. Table II shows the statistical data of each batch.

To compare the measurements made at different locations with different facilities, we did a statistical analysis of the critical strains obtained by GE and ASMC as shown in Table III. The results are consistent. 


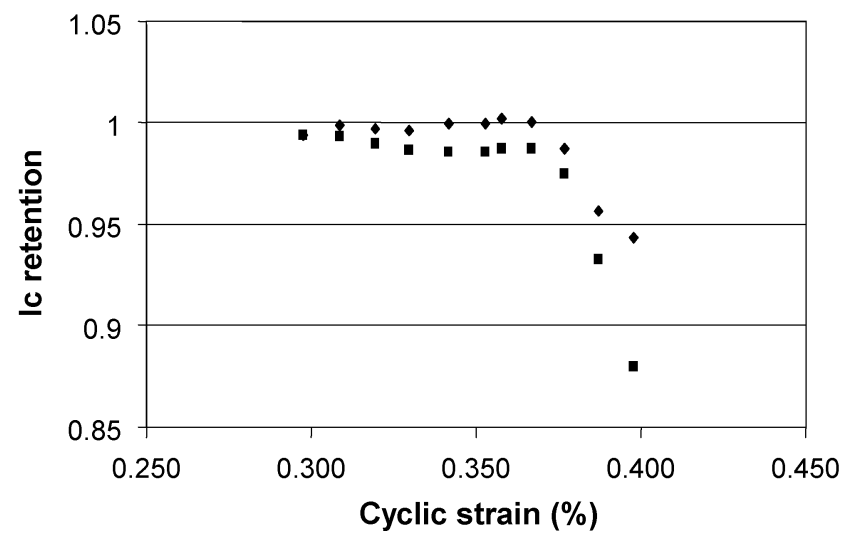

Fig. 4. A typical $\mathrm{I}_{\mathrm{C}}$ degradation as a function of tensile strain, the square dots are the data measured at the peak strain of the cycle, while the diamond dots were measured after the strain was relieved to zero.

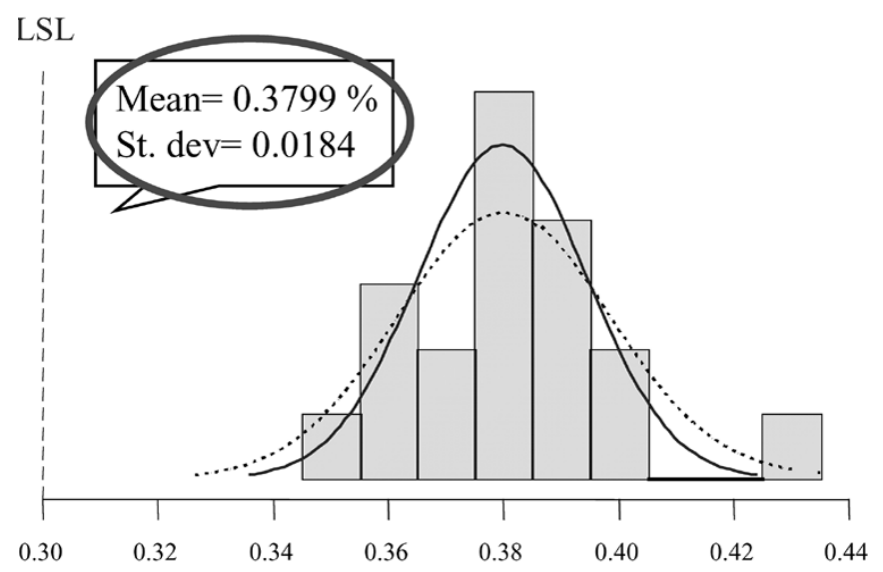

Fig. 5. Normal distribution of the critical strain.

TABLE II

BATCH-TO-BATCh VARIATION OF THE CRITICAL StRAins IN THE TENSILE TEST

\begin{tabular}{l|l|l|l}
\hline Batch number & $\mathrm{N}$ & Mean & StDev \\
\hline 3 & 5 & 0.3714 & 0.0117 \\
\hline 5 & 6 & 0.3853 & 0.0245 \\
\hline 16 & 5 & 0.3834 & 0.0118 \\
\hline 17 & 4 & 0.3760 & 0.0201 \\
\hline
\end{tabular}

TABLE III

COMPARISON OF THE MEASUREMENTS AT DifFERENT SiteS

\begin{tabular}{l|l|l|l}
\hline & $\mathrm{N}$ & Mean & StDev \\
\hline AMSC & 7 & 0.3841 & 0.0109 \\
\hline GE & 18 & 0.3810 & 0.0179 \\
\hline
\end{tabular}

\section{B. Compressive Strain}

Fig. 6 shows $I_{C}$ degradation observed during a typical repetitive run of compressive strain cycles. It is interesting to notice that the degradation is smaller at the peak compressive strain than that at the zero strain. Unlike the case of tensile cycling, $\mathrm{I}_{\mathrm{c}}$ reduction is observed even at small strains of $0.08 \%$. The degradation becomes larger with increasing strain. Saturation with cycle number of $I_{C}$ was seen at higher compressive strain levels (to at least $0.2 \%$ compressive). Putting all the measurement data into GE's DFSS toolset, we can build a transfer func-

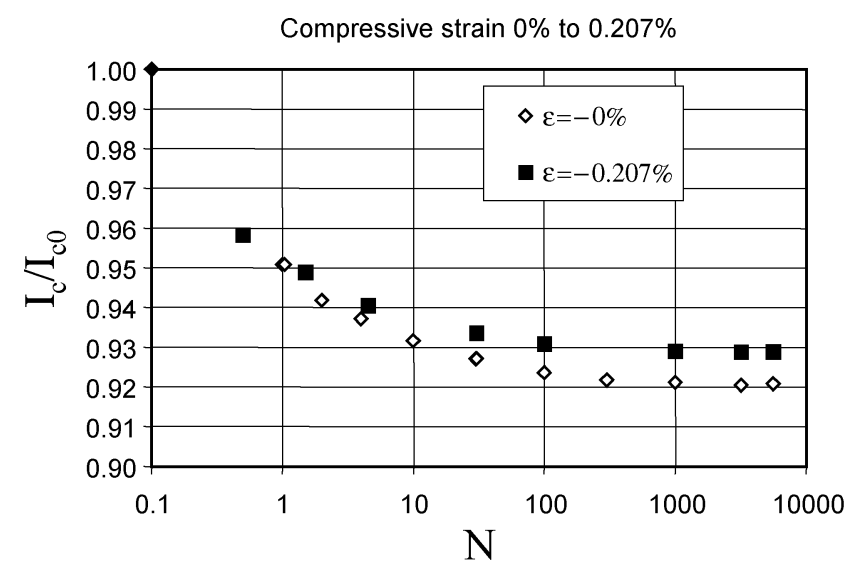

Fig. 6. Normalized Ic as a function of the number of cycles with a cyclic compressive strain of $-0.207 \%$.

TABLE IV

THE PREDICTEd COMPRESSIVE STRAIN FOR 95\% I RETENTION

\begin{tabular}{l|l|l|l}
\hline & & 100 cycles & 10000 cycles \\
\hline $\begin{array}{l}\text { Pred. Strain } \\
\text { for 95\% Ic } \\
\text { retention }\end{array}$ & Mean & $-0.163 \%$ & $-0.151 \%$ \\
\cline { 2 - 4 } & St. Dev. & $0.016 \%$ & $0.015 \%$ \\
\hline
\end{tabular}

tion between the critical compressive strain for a specific $\mathrm{I}_{\mathrm{c}}$ retention at different cycle numbers, and this transfer function is:

$$
\begin{aligned}
\varepsilon_{c}=5.324-12.8994\left(\frac{I_{c}}{I_{c 0}}\right)+7.476\left(\frac{I_{c}}{I_{c 0}}\right)^{2} \\
+0.00835 \ln (\mathrm{N})-0.006645 \ln ^{2}(\mathrm{~N}),
\end{aligned}
$$

where $\varepsilon_{\mathrm{c}}$ is the critical strain for $\left(\mathrm{I}_{\mathrm{c}} / \mathrm{I}_{\mathrm{c} 0}\right)$ retention, and $\mathrm{N}$ is the number of cycles. Substituting 0.95 for $\mathrm{I}_{\mathrm{c}} / \mathrm{I}_{\mathrm{c} 0}$ in the transfer function, we obtain the predicted strain for $95 \%$ Ic retention at 100 cycles and 10000 cycles.

Table IV gives the predicted critical strains and their standard deviations.

\section{Cycling at Room Temperature (Tensile)}

Spin tests are carried out to balance a rotor at room temperature during rotor assembly. Tensile strain results from the centrifugal loading. It is therefore important to know if strain cycles at room temperature affect the HTS wire performance.

Room temperature cyclic testing was carried out at GE. Samples were cycled 100 times to a fixed strain, the strain was then increased in $0.01 \%$ steps and the cycles repeated until $95 \% \mathrm{I}_{\mathrm{c}}$ retention was observed. An $\mathrm{I}_{\mathrm{c}}$ measurement was made initially and then after every 100 cycles. It was observed that the mean critical strain at room temperature is $0.254 \%$, which is smaller than the $0.38 \%$ observed at liquid nitrogen temperature, and the standard deviation is $0.020 \%$ (Fig. 7).

\section{CONCLUSION}

1. The fatigue behavior of $I_{c}$ of BSCCO HTS wire was investigated under tensile and compressive load at liquid nitrogen temperature and at room temperature. 


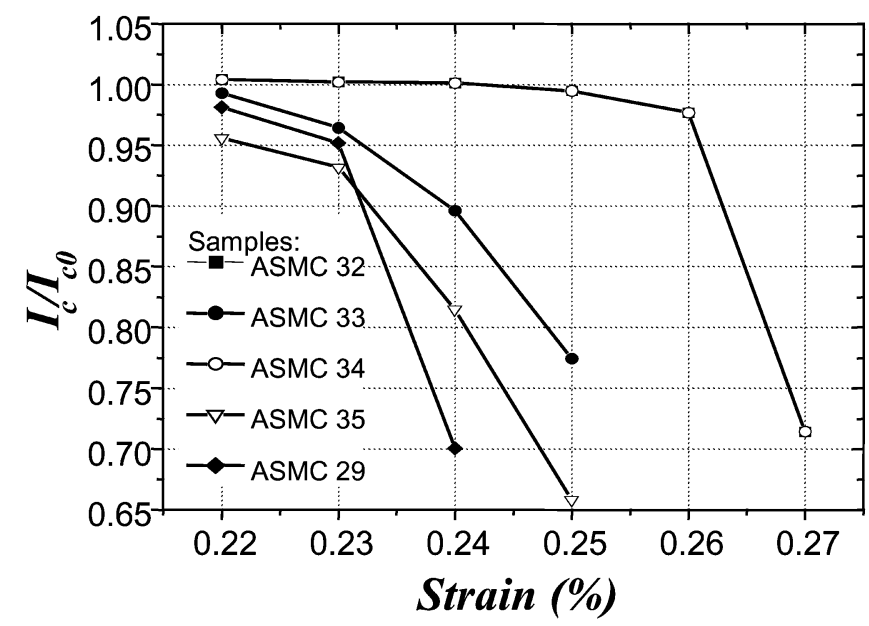

Fig. 7. Ic degradation as a function of tensile strain tested at room temperature. Samples were cycled 100 times at a fixed strain.

2. The measurements that were carried out at three different locations with different test facilities gave consistent results.

3. The $I_{c}$ degradation is less than $5 \%$ after

a) 100 cycles of 0 to $0.35 \%$ tensile strain or 0 to $0.2 \%$ compressive strain; and

b) 10000 cycles of 0 to $0.21 \%$ tensile strain or 0 to $0.15 \%$ compressive strain.
4) A transfer function for compressive regime was developed from the data obtained in the compressive cyclic tests. It was used to determine critical compressive strain for $95 \%$ Ic retention at $10^{2}$ and $10^{4}$ cycles.

5) For tensile strains, a dramatic degradation is observed around $0.36 \%$. This suggests that margin should be kept in tensile strain for application design.

6) Ic degradation saturated by 100 cycles in the strain range between $-0.2 \%$ and $0.35 \%$. Therefore, to characterize the Ic performance at $10^{4}$ cycles or higher, a 100 cycle test is sufficient.

\section{REFERENCES}

[1] B. ten Haken, R. B. Wilson, H. H. J. ten Kate, and J. Tenbrink, "Critical current reduction due to strain in Bi-2212 wires," in Applied Superconductivity, H. C. Freyhardt, Ed, Germany: DGM Informationsgellschaft $\mathrm{mbH}, 1993$, p. B235.

[2] B. ten Haken, H. H. ten Kate, and J. Tenbrink, "Compressive and tensile axial strain reduced critical currents in Bi-2212 conductors," IEEE Trans. Appl. Supercond., vol. 5, no. 2, pp. 1298-1301, 1995.

[3] J. W. Ekin, D. K. Finnemore, Q. Li, J. Tenbrink, and W. Carter, "Effect of axial strain on the critical current of Ag-sheathed Bi-based superconductors in magnetic fields up to 25 T," Appl. Phys. Lett., vol. 61, pp. 858-860, 1992.

[4] H. S. Shin and K. Katagiri, "Influence of external reinforcement on stress/strain characteristics of critical current in Ag/BSCCO superconducting tapes," in Pro. Int. Workshop Mechano-Electromagnetic Property of Composite Superconductor, Knob, 2001, pp. 31-34. 